

\title{
Morphodynamic modeling of erodible laminar channels
} Olivier Devauchelle, Christophe Josserand, Pierre-Yves Lagrée, Stéphane Zaleski

\section{To cite this version:}

Olivier Devauchelle, Christophe Josserand, Pierre-Yves Lagrée, Stéphane Zaleski. Morphodynamic modeling of erodible laminar channels. Physical Review E: Statistical, Nonlinear, and Soft Matter Physics, 2007, 76 (5), pp.056318. 10.1103/PhysRevE.76.056318 . hal-01449840

\section{HAL Id: hal-01449840 https://hal.sorbonne-universite.fr/hal-01449840}

Submitted on 12 Apr 2017

HAL is a multi-disciplinary open access archive for the deposit and dissemination of scientific research documents, whether they are published or not. The documents may come from teaching and research institutions in France or abroad, or from public or private research centers.
L'archive ouverte pluridisciplinaire HAL, est destinée au dépôt et à la diffusion de documents scientifiques de niveau recherche, publiés ou non, émanant des établissements d'enseignement et de recherche français ou étrangers, des laboratoires publics ou privés. 


\title{
Morphodynamic modeling of erodible laminar channels
}

\author{
Olivier Devauchelle, Christophe Josserand, \\ Pierre-Yves Lagrée and Stéphane Zaleski \\ Institut Jean le Rond d'Alembert, Université Pierre et Marie Curie - Paris 6, \\ CNRS-UMR 7190, Case 162, 4 place Jussieu, 75252 Paris Cédex 05-France
}

\begin{abstract}
A two dimensional model for the erosion generated by viscous free-surface flows, based on shallowwater equations and the lubrication approximation is presented. It has a family of self-similar solutions for straight erodible channels of invariant section, with an aspect ratio that increases in time. It is also shown, through a simplified stability analysis, that a laminar river can generate various bar instabilities very similar to those observed in natural rivers. This theoretical similarity reflects the meandering and braiding tendencies of laminar rivers indicated by F. Metivier, and P. Meunier, (Journal of Hydrology, 271, pp 22-38 (2003)). Finally, we propose a simple scenario for the transition between patterns observed in experimental erodible channels.
\end{abstract}

PACS numbers: 


\section{INTRODUCTION}

Natural rivers seldom form straight beds. Instead, they usually develop braids or meanders as a consequence of current-induced sediment transport. The understanding of such river sedimentation mechanisms can also help to characterize the spatial heterogeneity of alluvial rocks, which is a key parameter when simulating aquifer flows or oil traps in petroleum reservoirs [1]. The theoretical work of [2], [3] and [4] introduced among geomorphologists the fruitful idea that such patterns may originate in the linear instability of the flow, bed and bank system. Two-dimensional turbulent shallow water equations associated with a simple sediment transport law are able to predict the formation of alternate bars in channels of constant width. Such bars have been commonly accepted as a key phenomenon for braids and meander formation [5]. Numerous refinements of this theory may be found in the literature: [6] performed the bar stability analysis in three dimensions, while [5] focused on the differentiation between braids and meanders. Later [7] and [8] relaxed the rigid-banks hypothesis. [9] and more recently [10] modelled the non-linear evolution of free bars. All these works (and to our knowledge, every study in this field) considered turbulent flows, which is entirely legitimate as far as natural rivers are concerned (the average Reynolds number of the Seine river in Paris is about $10^{6}$ ). However, one should not conclude from this ubiquity of turbulence that braiding and meandering are inherently turbulent phenomena. [11] very recently accumulated experimental evidences showing that laminar flumes may generate many patterns created by real rivers. In particular, the constant flow of a thin liquid film down an homogeneous granular bed initially crossed by a straight channel exhibits rather complex pattern dynamics as the flume is deformed by erosion (see [12]). First, the channel widens while remaining straight. Then a meander-like instability develops, which deforms both the bed and the banks. Eventually, more bars develop in the middle of the channel and the river starts to braid[32]. This behavior is qualitatively comparable to the one of larger channels, at higher Reynolds number (see the two meters-wide experiment of [13]). To our knowledge, no quantitative experimental results have been published about river erosion instabilities in the laminar regime. As a consequence, the results presented here can only be compared to the qualitative evolution described in [12]. Reference to turbulent experiments can only illustrate the sound similarity with the laminar case.

Our objective is to comfort the idea that micro-rivers can be an intermediate step toward 
the understanding of natural rivers morphodynamics. We do not claim that quantitative results from micro-rivers could be extrapolated to field results (see section II A). We are rather convinced that such small-scale experiments share with larger ones many features still under investigation (non-linearity of the flow-sediment interaction, equilibrium shape of the bed, behavior and influence of the bank). Such laminar flow approach can also help to disentangle the role of the turbulence in the river morphodynamic. Moreover, theoretical as well as numerical river models could be easily tested against micro-rivers data, before adding the complexity of turbulence and switching to larger experiments and natural rivers.

In a first section, a two-dimensional evolution model for laminar flumes is presented. It is based on the assumption that the velocity profile is close to Nußelt's one. A rather general erosion law is then discussed and compared to the experiments of [14]. The following section is devoted to the study of a straight river widening process, and an analytical solution is proposed in a simple case. In the third section, the linear stability analysis of a straight laminar flume with solid banks is presented.

\section{A TWO-DIMENSIONAL MODEL}

Let us consider an experiment during which an initial channel incised into a uniform and non-cohesive sand layer is eroded by a viscous flow. If the slope of the sand bed remains small enough, one may use two-dimensional equations to model both the water flow and the sediment transport. A rather general assumption (commonly used in river mechanics) consists in the time-scale separation between the flow and erosion process: the bed evolves slowly enough for the flow to be quasi-static (see [5], [4], [6] and [8]). Of course, this hypothesis fails during such violent events as roll-waves .

In the present article the following notations are used (see also figure 1):

- $x$ and $y$ are the coordinates in the plane of the experiment, the first aiming toward the main slope. $z$ is the coordinate normal to the plate;

- $h$ is the elevation of the sand surface and $d$ is the water depth $(\eta=h+d$ is thus the water level);

- $\mathbf{u}=(u, v)$ is the vertically averaged water velocity, the horizontal water flux components being $u d$ and $v d$; 


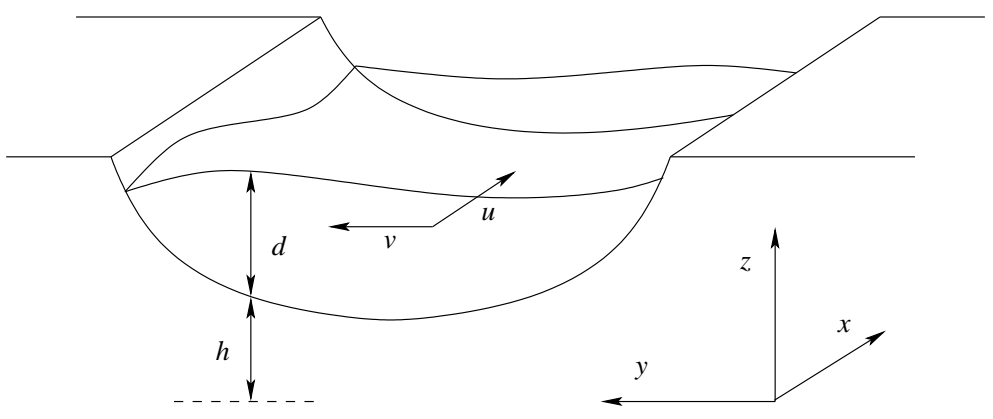

FIG. 1: Sketch of a riverbed.

- $S$ is the plate tilt;

- $g$ is the magnitude of gravity, and $\nu$ is the kinematic viscosity of water.

\section{A. Water flow}

The present micro-river model requires that the water flow is laminar, so that it can be approximated by a vertical velocity profile of Nußelt type. For this assumption to hold, the Reynolds number $R e=u_{0} d_{0} / \nu$ must remain low enough $\left(d_{0}\right.$ and $u_{0}$ are the typical height and velocity scales respectively). The water velocity $\widehat{\mathbf{u}}$ is thus approached by a parabolic velocity profile which adapts instantaneously to the topography:

$$
\widehat{\mathbf{u}}(x, y, z, t) \approx \frac{3}{2} \xi(2-\xi)(u(x, y, t), v(x, y, t), 0),
$$

where $\xi=(z-h) / d$. This mehtod corresponds to the lubrication approximation. Different approaches may be found in [15] or [16], though in one dimension. Secondary currents are thus neglected, although many authors believe they strongly influence erosion in developed meanders (see for example [17]). The effect of secondary currents is sometimes taken into account in the Saint Venant frame by mean of a ad hoc term in sediment transport equations (see [8]). Since the present study is restricted to straight channels, we will hereafter assume that the curvature of the flow remains small enough for the secondary currents to remain negligible (this argument is developed by [18]). This approximation is actually correct for any curvature, provided the Reynolds number is low enough.

The vertical integration of the Navier-Stokes equations, associated with (1), leads to the 
viscous shallow water equations:

$$
\begin{gathered}
\frac{6}{5}(\mathbf{u} \cdot \nabla) \mathbf{u}=g\left(-\nabla(d+h)+S \mathbf{e}_{x}\right)-\frac{3 \nu}{d^{2}} \mathbf{u}, \\
\nabla \cdot(\mathbf{u} d)=0,
\end{gathered}
$$

where $\mathbf{e}_{x}$ is the unit vector parallel to the $x$-axis. These equations are very similar to those used for turbulent rivers. The only differences lie in the coefficient $6 / 5$ which becomes 1 in the turbulent case, and in the friction term $-3 \nu \mathbf{u} / d^{2}$ which becomes $-C_{f}\|\mathbf{u}\| \mathbf{u} / d\left(C_{f}\right.$ is

the Chézy friction coefficient). One cannot thus expect micro-rivers to be scaled models for natural ones, since the laminar flow equations cannot be reduced to the classical turbulent ones. On the other hand, it is interesting to point out similarities and differences between these two different (although not too far) cases, turbulent and laminar.

\section{B. Sediment transport}

The river bed evolves under the influence of both erosion and avalanches. In the present context erosion consists of flow-induced bed-load transport of sand grains. On the other hand, avalanches are collective phenomena triggered by an excess slope of the sand surface. In the continuous model developed here, we can only handle the average effects of erosion and avalanches. This approximation allows for the definition of a total volumic sediment flux $\mathbf{q}(x, y, t)$ integrated along the vertical direction. Assuming a strong time scale separation between erosion and avalanches, one may consider the associated fluxes (respectively $\mathbf{q}_{e}$ and $\mathbf{q}_{a}$ ) as independent. The continuity equation for sand then reads:

$$
\frac{\partial h}{\partial t}=-\nabla \cdot \mathbf{q}
$$

where $\mathbf{q}=\mathbf{q}_{e}+\mathbf{q}_{a}$. Finally, closure relations have to be deduced, either on dimensional, physical or empirical grounds in order to link (4) to the flow equations.

Erosion contribution. Most of the relations between the sediment flux and the flow are proposed in the literature as functions of the Shields number $\theta$, which expresses the ratio between hydrodynamic forces exerted on a grain to its apparent weight :

$$
\theta=\frac{|\tau|}{d_{s}\left(\rho_{g}-\rho_{w}\right) g}
$$


where $d_{s}, \rho_{w}, \rho_{g}$ and $\tau$ are respectively the typical particle diameter, the density of water, the density of a grain and the bottom shear stress. As suggested by [19], we propose the following expression as a classical relationship (see the review of [20]) for small slope:

$$
\mathbf{q}_{e}=\phi(\theta)\left(\frac{\tau}{|\tau|}-\mathbf{G} \cdot \nabla h\right)
$$

where $\phi$ is a growing function that may include a threshold value, and $\mathbf{G}$ is a diagonal operator.

To determine a plausible form for $\phi(\theta)$ we shall use recent experimental results obtained by Charru, Mouilleron and Eiff for grain transport in the viscous flow regime[14]. Their results on grain transports are partially reproduced on figure 2 and these authors suggest then the following transport law :

$$
\frac{N_{p} d_{s}^{2}}{V_{s}}=0.85 \theta(\theta-0.12) \mathcal{H}(\theta-0.12)
$$

where $N_{p}$ is the particle flux, and $V_{s}$ is the Stokes settling velocity of a particle $\left(V_{s}=\right.$ $\left.\left(\rho_{s}-\rho_{w}\right) d_{s}^{2} /\left(\rho_{w} \nu\right)\right) . \mathcal{H}$ is the Heavyside function. $N_{p}$ is linked to $q$ through $q=N_{p} \mathcal{V}$, where $\mathcal{V}$ is the volume filled by a particle in the sediment layer. According to this expression, no sediment is transported at Shields number values below a threshold. However, [14] indicates that some particles remain in motion at Shields numbers lower than 0.12 during a transition regime, and will eventually settle after an "armoring time [...] very large compared to the hydrodynamic time scales". Maybe due to this armouring time, their measurements of the sediment flux do not exactly vanish below the threshold (see figure 2). It is thus tempting, as already proposed earlier to model sediment transport under turbulent flow (such as [10] for instance) to use a pure power law functional instead of formula (7). Such a law may be adjusted to fit the data of [14] (see again figure 2) and it gives:

$$
\frac{N_{p} d_{s}^{2}}{V_{s}}=5.13 \theta^{3.75}
$$

Relations (7) and (8) cannot be in fact clearly separated by the experiments of [14]. Thus, for simplicity reasons, we will use the second one in what follows. This choice will be discussedd again in sections III and IV. The general form of the erosion law is then taken as:

$$
\phi(\theta)=\phi_{0} \theta^{\beta}
$$

reminding that $\phi_{0} \approx 5.13 \mathcal{V} V_{s} / d_{s}^{2}$ and $\beta \approx 3.75$ in [14]. 
The second term in (6) reproduces the slope-induced deviation of the sediment flux. [10] sets $\mathbf{G}=\gamma \mathbf{I}$ where $\gamma$ is a constant of order one. This isotropic approximation is questionable, but should not influence qualitatively the results. This term is mathematically essential to cut-off short wavelength instabilities (see Section IV B). In the frame of the laminar SaintVenant approximation, the above sediment transport equation becomes then

$$
\mathbf{q}_{e}=E_{e}\left(\frac{|\mathbf{u}|}{d}\right)^{\beta}\left(\frac{\mathbf{u}}{|\mathbf{u}|}-\gamma \nabla h\right),
$$

with $E_{e}=\phi_{0}\left(3 \rho_{w} \nu /\left((2 r)\left(\rho_{g}-\rho_{w}\right) g\right)\right)^{\beta}$.

Avalanches. The full dynamics of avalanches is far out the scope of this study. Instead, we may propose a simple model which reproduces the following features:

- the sand mass is conserved through the avalanche process;

- there are no avalanches under a critical slope $\alpha$;

- above the critical angle, $\mathbf{q}_{a}$ is directed toward the main slope and increases with the slope value.

Considering these criteria, we propose the following expression :

$$
\mathbf{q}_{a}=-E_{a} \mathcal{F}(|\nabla h|) \frac{\nabla h}{|\nabla h|},
$$

where $\mathcal{F}(\cdot)=(\cdot-\alpha) \mathcal{H}(\cdot-\alpha)$ and $E_{a}$ is a constant. Indeed, similar law has been successfuly employed for eolian dunes by [21].

Finally, it is important to notice here that these fluxes $\mathbf{q}_{e}$ and $\mathbf{q}_{a}$ do not account for the saltating grain dynamics. In a simplified approach, the grains motions would end up into a settling distance at which the fluxes developp (see [22] and references herein for a discussion of these terms). It manifests in the dynamics through a phase shift between the shear stress and the fluxes. By sake of simplicity, we do not take into account such a term although it could be implemented easily. Such approximation corresponds somehow to a limit where the density ratio between grains and water is high. In the following, it is in fact remarkable that the instability exists without such phase shift. 


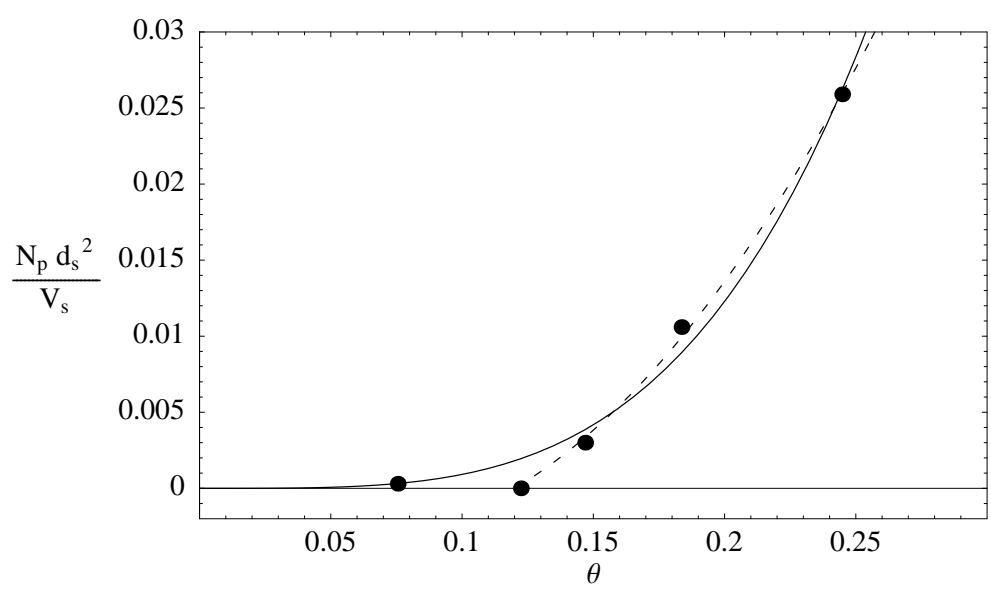

FIG. 2: Different transport laws compared with the experimental results obtained by [14]. The grains are transported by a viscous flow in a circular Hele-Shaw cell. $N_{p}$ is the particule flux, and $V_{s}$ is the settling velocity of a particle. Dashed : threshold law proposed by [14], $N_{p} d_{s} / V_{s}=$ $0.85 \theta(\theta-0.12) \mathcal{H}(\theta-0.12)$. Solid line : power-law fit, $N_{p} d_{s} / V_{s}=5.13 \theta^{3.75}$.

\section{Boundary conditions}

Flow equations (2) and (3) together with sediment transport equations (4), (10) and (11) form a closed system. To solve this system in the fixed domain $\Omega$, conditions must be specified on its boundary $\partial \Omega$. Their general form writes

$$
\lambda_{u} d+\mu_{u} \mathbf{u} \cdot \mathbf{n}=\pi_{u}, \quad \lambda_{h} h+\mu_{h} \mathbf{q} \cdot \mathbf{n}=\pi_{h},
$$

where $\lambda_{u}, \mu_{u}, \pi_{u}, \lambda_{h}, \mu_{h}$ and $\pi_{h}$ are functions to be specified. $\mathbf{n}$ is the $2 \mathrm{D}$ unit vector normal to $\partial \Omega$, aiming outward. In the general case, $\Omega$ may include sub-domains where $\mathbf{q}=0$. In such domains, the evolution equation becomes $\partial h / \partial t=0$.

If one wants to restrict the analysis to the active sub-domain $\Omega_{+}(t)$ where $\mathbf{q} \neq 0$, the conditions to be imposed on its mobile boundary $\partial \Omega_{+}(t)$ are

$$
\begin{aligned}
\mathbf{u} \cdot \mathbf{n} & =0, \quad \mathbf{q}_{+} \cdot \mathbf{n}=c\left(h_{+}-h_{-}\right) \\
\frac{\mathrm{d} h_{+}}{\mathrm{d} t}+\frac{\mathrm{d} h_{-}}{\mathrm{d} t} & =-\nabla \cdot \mathbf{q}_{+}+c\left(\mathbf{n} \cdot \nabla h_{+}+\mathbf{n} \cdot \nabla h_{-}\right) .
\end{aligned}
$$

In the above equations, $c$ is the normal velocity of the $\partial \Omega_{+}(t)$, the subscripts + and denotes quantities evaluated respectively inside and outside $\Omega_{+}(t)$. d/d $t$ is the convective 
derivative at a point of $\partial \Omega_{+}(t)$ moving with velocity $c \mathbf{n}$. The first boundary condition reflects the time scale separation between flow and erosion (so that the condition for the normal velocity of the boundary is zero instead of $c$ ). The following ones correspond to the sediment mass conservation equations integrated over a small domain crossed by $\partial \Omega_{+}(t)$. A special case of (13) has been derived by [23].

The classical conditions for non-erodible and impermeable banks are obtained from (13) by setting $c=0$. In that case, and for turbulent flow, bar instabilities may develop (see [10] for stability analysis and weakly non-linear theory of bars). The present paper shows that bar instabilities of the same nature may also develop in laminar conditions. To switch from bars to meanders and braids, the condition $c=0$ must be relaxed. In a seminal paper, [8] used an empirical estimation of $c$ as a function of the additional stress induced by secondary flows. They also implicitly assumed that the bank material input due to erosion had no influence on the bed evolution (they $\operatorname{set} \mathbf{q}_{+} \cdot \mathbf{n}=0$ despite a finite value for $c$ ). They showed that meandering results from the interaction between alternate bars instabilities and the so-called bend instability, which results from the curvature of the bank. For micro-rivers, their hypothesis would not hold, since bed and banks are of the same granular material. The elaboration of a bank evolution law able to model the effect of avalanches is the subject of on-going work. The present stability analysis (section IV) is restricted to channels with rigid banks $(c=0)$, as were the first equivalent studies in the case of real rivers (see [4]). On the other hand, in the case of a prismatic river (section III), equations are solved on the whole $\Omega$ domain, and thus no boundary conditions are required. For the full determination of the solution moreover one has to prescribe global boundary conditions on the upstream and downstream fluxes of water.

\section{PRISMATIC CHANNELS}

For a straight, $x$-invariant river, the equations derived in section II turn into a one dimensional non-linear diffusion equation which admits self-similar solutions. The reader interested in the problem of real turbulent river cross-section, a complex two-dimensional problem in the general case, may refer to [23-27] among others. 


\section{A. A non-linear diffusion equation}

For a prismatic river, any quantity only depends on time and the transverse coordinate $y$. The flow equations (2) and (3) thus become

$$
u(y, t)=\frac{g S}{3 \nu} d(y, t)^{2}, \quad v=0, \quad d(y, t)+h(y, t)=\eta(t) .
$$

The water discharge

$$
Q_{w}=\int_{-\infty}^{\infty}(u d) \mathrm{d} y
$$

is usually fixed in experiments, and thus governs the evolution of $\eta(t)$. For the sake of simplicity, we will consider a different case in what follows. If $\eta$ instead is fixed, this arbitrary constant may be set to zero (and thus $h=-d$ ). This case represents a river supplied by an infinite reservoir. The sediment transport equations (4), (10) and (11) lead to

$$
\frac{\partial h_{*}}{\partial t_{*}}=\frac{\partial}{\partial y_{*}}\left(\left(-h_{*}\right)^{\beta} \frac{\partial h_{*}}{\partial y_{*}}\right)-\frac{1}{\epsilon_{a}} \frac{\partial}{\partial y_{*}}\left(F\left(\left|\frac{\partial h_{*}}{\partial y_{*}}\right|\right) \operatorname{sign}\left(\frac{\partial h_{*}}{\partial y_{*}}\right)\right) .
$$

In the above equation, the starred quantities are dimensionless. The initial depth $d_{0}$ is the characteristic length.

$$
T=\frac{d_{0}^{2}}{\gamma \phi_{0}}\left(\frac{d_{s}\left(\rho_{g}-\rho_{w}\right)}{\rho_{w} S d_{0}}\right)^{\beta}
$$

is the typical widening time scale. $\epsilon_{a}=d_{0}^{2} /\left(T E_{a}\right)$ is a non-dimensional number which compares typical avalanches flux to erosion ones. It will be considered very small in what follows.

The non-linear diffusion equation (16) may be solved numerically. A classical first-order finite-volume scheme leads to the solution presented in figure 3 at different times. The initial width is $w_{*, 0}=2.5$, and $\epsilon_{a}$ is fixed to 0.1 . The value of $\epsilon_{a}$ has a weak influence on the result, provided it is small (the same computation performed with $\epsilon_{a}=0.01$ gives similar results). However, the Courant, Friedrichs, and Lewy condition imposes a numerical time step smaller and smaller as the value of $\epsilon_{a}$ is reduced. The erosion law is fixed by setting $\beta=3.75$. The influence of any other parameter of the problem, such as the Froude number or the channel slope is embedded in the definition of the time and space scales.

Trough erosion, the river widens and gets shallower, while its cross-section area remains constant. This is in qualitative accordance with experiments during which the water outflow was fixed, instead of the water level in the present theory (see [12], [28], [7] and [29]). Since the erosion law (8) presents no threshold, this widening process will never stop in 
the frame of this model, which may seem unreasonable. Some authors ([7], [29]) managed to reach an equilibrium width, but in most experiments ([12], [13]) the channel invariance along the $x$-axis falls before any equilibrium can be reached, due to bar instabilities ([7] and [29] removed the meandering tendency by using a half-river). To our knowledge, no river-widening experiment where carried out in the laminar regime at a fixed water level.

\section{B. Self-similar solutions}

If avalanches are neglected, or if the transverse slope of the channel $\partial h / \partial y$ can remain always smaller than the critical slope $\alpha$ (so that no avalanche occurs), the last term of (16) drops. This particular case has simple self-similar solutions of the form

$$
h_{*}\left(y_{*}, t_{*}\right)=\frac{1}{t_{*}^{1 /(\beta+2)}} f(\chi) .
$$

where $\chi=y_{*} / t_{*}^{1 /(\beta+2)}$. Then (16) leads to

$$
\frac{\partial}{\partial \chi}\left((-f)^{\beta} \frac{\partial f}{\partial \chi}+\frac{\chi f}{\beta+2}\right)=0 .
$$

If $f_{s}$ is a symmetrical solution to (19), $\mathrm{d} f_{s} / \mathrm{d} \chi=0$ at $\chi=0$, and thus integration of (19) gives

$$
f_{s}(\chi)=\left\{\begin{array}{cl}
-\left(\frac{\beta}{A-2(\beta+2)} \chi^{2}\right)^{1 / \beta} & \text { if } \quad \chi \in\left[0, \sqrt{A \frac{2(\beta+2)}{\beta}}\right] \\
0 & \text { elsewhere }
\end{array}\right.
$$

where $A$ is a constant. Let $\mathcal{A}_{*}$ be the (non-dimensional) area of the cross-section. Then

$$
\mathcal{A}_{*}=\int_{0}^{\infty} h_{*}\left(y_{*}, t_{*}\right) \mathrm{d} y_{*}=-A^{1 / \beta+1 / 2} \int_{0}^{\sqrt{\frac{2(\beta+2)}{\beta}}}\left(\frac{\beta}{2(\beta+2)} \xi^{2}-1\right)^{1 / \beta} \mathrm{d} \xi .
$$

Thus when avalanches can be neglected, (16) admits a set of self-similar solutions parameterized by their cross-section area. The solution corresponding to $\mathcal{A}_{*}=-2.5$ is represented on figure 3. Despite its rectangular initial cross-section, the numerical solution converges towards its self-similar counterpart. This behavior seems quite general: it very weakly depends on the initial conditions or the value of $\beta$.

Only for $\beta=1$ (that is for an unrealistic linear erosion law) does the self-similar solution behave regularly at the banks. In that case, the river cross-section is a parabola. It width increases as $t^{1 / 3}$ while it shallows as $t^{-1 / 3}$. If the initial shape is flat enough to avoid 


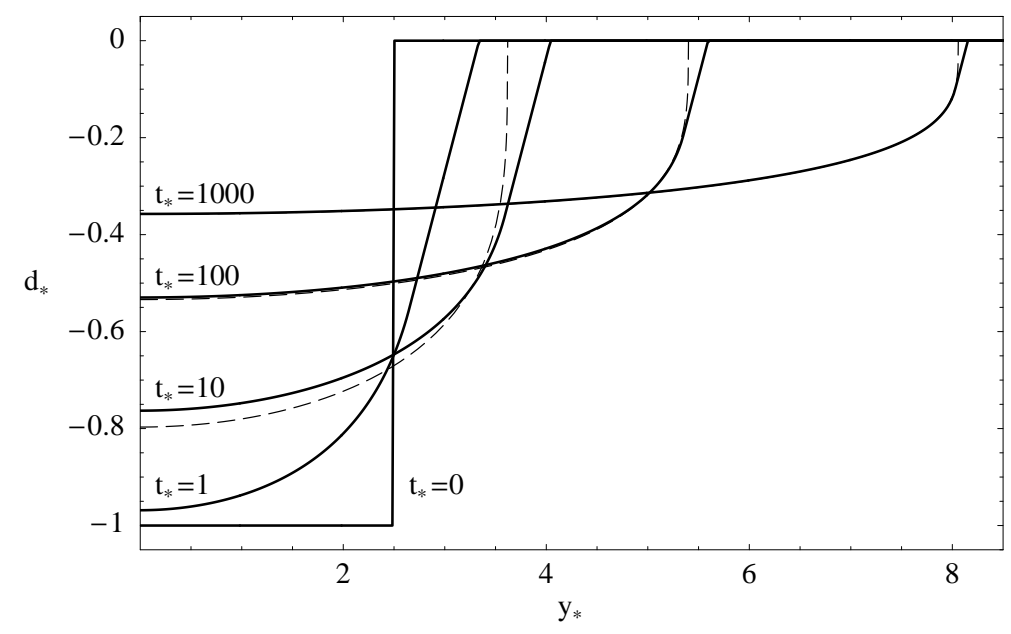

FIG. 3: Widening of a straight laminar channel through erosion, modelled with (16). Parameter values are $\epsilon_{a}=0.1, \alpha=0.8$, and $\beta=3.75$. Solid lines: numerical solutions of (16) at different times. Dashed line: self-similar solution (without avalanches, see section III B) at $t_{*}=10, t_{*}=100$ and $t_{*}=1000$. The presence of avalanches seems not to influence much the self-similar evolution of the profile.

avalanches, this condition holds at any time. Unfortunately this case cannot model erosion patterns formation, for it is unconditionally stable (see section IV).

On the other hand, if $\beta>1$ the picture is quite different. The continuous widening process still holds: the width increases as $t^{1 /(\beta+2)}$, while the depth decreases as $t^{-1 /(\beta+2)}$. However, in that case the bed slope $\mathrm{d} h_{*} / \mathrm{d} y_{*}$ diverges at the banks. Thus avalanches must occur at the banks, and the self-similar solution fails. This tendency is observed in laboratory experiments (see [30] among others), and was already pointed out by [23]. The effect of bank avalanches is uneasy to quantify analytically. According to numerical simulations in the case $\beta=3.75$ however (see figure 3), they do not seem to influence strongly the bed evolution far enough from the banks. Consequently one may still use the results of the self-similar theory as good approximations of true solutions.

\section{LINEAR STABILITY}

Experimental channels such as the one of [12] or [13] often remain stable for a while, then develop meanders which in turn are followed by more complex braided-like patterns. 
This scenario of transitions (sometimes called ageing) may be interpreted as the successive dominance of different unstable modes. If the widening process presented in the previous section is slow enough, a straight river may be chosen as a quasi static base state for a stability analysis. This is what we will assume in the following, so we will disregard any interaction between widening and instabilities.

\section{A. Derivation of the dispersion relation}

In order to present the simplest stability model which keeps the essential features of channel stability, we will consider a rectangular base state with solid-wall boundaries, of width $w_{0}$ and depth $d_{0}$ (its aspect ratio is thus $R=w_{0} / d_{0}$ ). The boundary conditions at the bank are $\mathbf{u} \cdot \mathbf{n}=0$ and $\mathbf{q} \cdot \mathbf{n}=0$. The basic water velocity is uniform and parallel to the $x$-axis $\left(u_{0}=g S d_{0}^{2} /(3 \nu)\right)$, and so is the basic sediment flux $\left(q_{0}=E_{e}\left(u_{0} / d_{0}\right)^{\beta}\right)$. Let us seek travelling-wave perturbations of this base state:

$$
\varphi(x, y, t)=\varphi_{0}+\epsilon \varphi_{*}\left(\frac{y}{w_{0}}\right) e^{i\left(k x / w_{0}-\omega t /(\gamma T R)\right)},
$$

where $\varphi=\left(u, v, h, d, q_{x}, q_{y}\right)$. The base state corresponds to $\varphi_{0}=\left(u_{0}, 0,-d_{0}, d_{0}, q_{0}, 0\right)$ and the perturbation is $\varphi_{*}=\left(u_{0} u_{*}, u_{0} v_{*}, d_{0} h_{*}, d_{0} d_{*}, q_{0} q_{x, *}, q_{0} q_{y, *}\right) . T=d_{0} w_{0} /\left(\gamma R q_{0}\right)$ is the characteristic erosion time defined in section III A, and $\epsilon$ is a small dimensionless amplitude of the perturbation. $k$ is a real dimensionless wavenumber whereas $\omega$ is complex in the general case. (2), (3), (10) and (4) lead to the following system:

$$
\begin{gathered}
\left(\frac{6}{5} F^{2} i k+R S\right) u_{*}+i k h_{*}+(i k-2 R S) d_{*}=0, \\
\left(\frac{6}{5} F^{2} i k+R S\right) v_{*}+\frac{\mathrm{d} d_{*}}{\mathrm{~d} y}+\frac{\mathrm{d} h_{*}}{\mathrm{~d} y}=0, \\
i k\left(d_{*}+u_{*}\right)+\frac{\mathrm{d} v_{*}}{\mathrm{~d} y}=0, \\
-i \omega h_{*}+i k q_{x, *}+\frac{\mathrm{d} q_{y, *}}{\mathrm{~d} y}=0, \\
q_{x *}=\beta u_{*}-\frac{i k \gamma}{R} h_{*}-\beta d_{*}, \quad q_{y *}=v_{*}-\frac{\gamma}{R} \frac{\mathrm{d} h_{*}}{\mathrm{~d} y} .
\end{gathered}
$$

In the above system, $F=u_{0} /\left(g d_{0}\right)^{1 / 2}$ is the Froude number of the unperturbed channel. Parameters $F, S$ and $R$ may be varied independently in experiments. Indeed, if $Q_{w}$ is the 
water outflow of the river, then

$$
w_{0}=R\left(\left(\frac{3 F \nu}{S}\right)^{2} \frac{1}{g}\right)^{1 / 3}, \quad Q_{w}=3 R\left(\frac{9 F^{8} \nu^{5}}{g S^{5}}\right)^{1 / 3}
$$

The full $(F, R, S)$-space may be explored by tuning the slope of the apparatus, the initial width of the channel and the water outflow. Of course, the validity of the present theory requires the parameters to satisfy some conditions. First, the flow has to be laminar. The low Reynolds number condition can be easily checked:

$$
R e=\frac{u_{0} d_{0}}{\nu}=\frac{3 F^{2}}{S}
$$

Capillarity can also cause the failure of the theory[33]. Near the banks of the channel, capillarity generates a meniscus of characteristic size $l_{c}\left(l_{c}=(\Gamma /(\rho g))^{1 / 2}\right.$, where $\Gamma$ is the surface tension). The quantity of water flowing through the meniscus zone should remain negligible as compared to the total outflow. As a crude approximation, the outflow in the meniscus zone $Q_{w, m}$ is evaluated by Poiseuille's formula : $Q_{w, m} \sim g S l_{c}^{4} / \nu$. The condition $Q_{w} \gg Q_{w, m}$ thus reads

$$
R \gg\left(\frac{l_{c}}{d_{0}}\right)^{4}
$$

The ratio of the water depth versus the capillary length is given by

$$
\frac{d_{0}}{l_{c}}=3^{2 / 3} g^{1 / 6}\left(\frac{\rho}{\Gamma}\right)^{1 / 2}\left(\frac{F \nu}{S}\right)^{2 / 3} .
$$

Consequently, (30) may be satisfied for any values of $R, F$ and $S$ provided the viscosity of the fluid is high enough[34]. Typical parameters values during the experiment of [12] (carried on with pure water) are $Q_{w}=13 \cdot 10^{-6} \mathrm{~m}^{3} \mathrm{~s}^{-1}, S=0.088$ and $w_{0}=0.1 \mathrm{~m}$. The non-dimensional number of the experiment thus are $R \approx 130, F \approx 2, R e \approx 130$ and $d_{0} / l_{c} \approx 0.3$. (30) was not satisfied in this experiment. However, the error resulting from this failure should only affect the evaluation of non-dimensional parameters from the experimental data, but the qualitative behavior predicted by the theory should hold.

Equations (23) to (27) may be reduced to

$$
\begin{gathered}
\frac{\mathrm{d}^{4} h_{*}}{\mathrm{~d} y^{4}}+A \frac{\mathrm{d}^{2} h_{*}}{\mathrm{~d} y^{2}}+B h=0, \\
\frac{\mathrm{d}^{3} h_{*}}{\mathrm{~d} y^{3}}\left(\frac{1}{2}\right)=\frac{\mathrm{d} h_{*}}{\mathrm{~d} y}\left(\frac{1}{2}\right)=\frac{\mathrm{d}^{3} h_{*}}{\mathrm{~d} y^{3}}\left(-\frac{1}{2}\right)=\frac{\mathrm{d} h_{*}}{\mathrm{~d} y}\left(-\frac{1}{2}\right)=0 .
\end{gathered}
$$


In the above equation,

$$
\begin{aligned}
& A=\left(36 F^{4} k^{3} \gamma+30 F^{2} k\left(-2 k^{2} \gamma-i k R(1+\beta+4 S \gamma)+i R \omega\right)+\right. \\
&\left.25 R S\left(2 i k^{2} \gamma+k R(-3+\beta-3 S \gamma)+R \omega\right)\right) /\left(5\left(6 F^{2} k-5 i R S\right) \gamma\right), \\
& B=\frac{1}{\gamma}\left(k \left(k^{3}\left(\gamma-\frac{6 F^{2} \gamma}{5}\right)+i k^{2} R(2 \beta+3 S \gamma)+\right.\right. \\
&\left.\left.\frac{1}{5} i\left(-5+6 F^{2}\right) k R \omega+3 R^{2} S \omega\right)\right) .
\end{aligned}
$$

Let $s$ be a solution of the characteristic equation attached to (32). Applying the boundary conditions leads to $s=i n \pi$, where $n$ is an integer. Finally, one may derive the dispersion relation from $(32)$ :

$$
\begin{array}{r}
\omega=\left(5 k R\left(5 i R S\left(-n^{2} \pi^{2}(-3+\beta)+2 k^{2} \beta\right)-6 F^{2} k\left(2 k^{2} \beta+n^{2} \pi^{2}(1+\beta)\right)\right)-\right. \\
\left.i\left(k^{2}+n^{2} \pi^{2}\right)\left(6 F^{2} k-5 i R S\right)\left(\left(-5+6 F^{2}\right) k^{2}-5 n^{2} \pi^{2}-15 i k R S\right) \gamma\right) / \\
\left(R\left(6 F^{2} k-5 i R S\right)\left(\left(-5+6 F^{2}\right) k^{2}-5 n^{2} \pi^{2}-15 i k R S\right)\right) .
\end{array}
$$

\section{B. Results interpretation}

The linear stability of a channel depends on the sign of the maximum growth rate over $n$ and $k$, respectively the transverse and longitudinal wavenumbers. We will thus focus on the imaginary part $\sigma$ of $\omega$ in what follows. Let $\sigma_{m}$ be the maximum growth rate, and $k_{m}$ and $n_{m}$ the corresponding wavenumbers (i.e. $\sigma_{m}=\sigma\left(k_{m}, n_{m}\right)=\max _{k \in \mathbb{R}, n \in \mathbb{N}}(\sigma)$ ). The transverse wavenumber $n$ characterizes the instability pattern: $n=0$ for $y$-invariant dunes (this mode can also initiate step-pool instability), $n=1$ for meanders and $n>1$ for braided patterns. The present theoretical frame fails to predict the step-pool instability often observed in narrow channels [31], as $\sigma$ is always negative for $n=0$. This is not surprising for the phase-shift between the bed deformation and the water shear stress is neglected here (this phase shift controls sand ripple formation for instance [22]). For higher modes, on the other hand, a positive growth rate is possible (see figure 4), despite the lubrication approximation. This indicates that the instability mechanism governing bars formation is different than the phase shift induced by the advection term in the case of dunes and ripples.

The fluid and sediment choices determine parameters $\gamma$ and $\beta$. Both parameters are crucial to the present model. The diffusion term which is proportional to $\gamma$ stabilizes the 


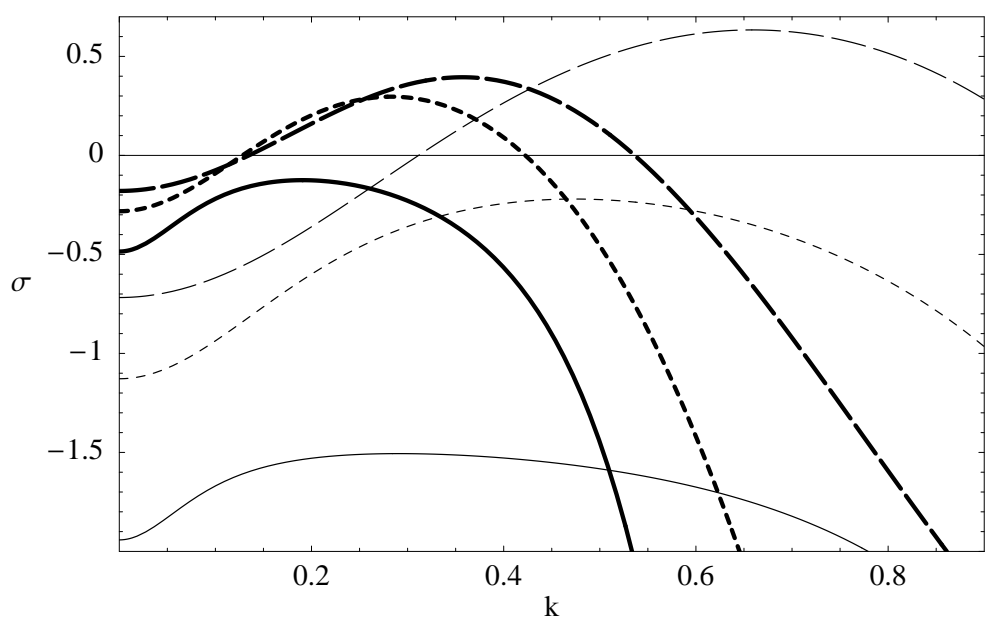

FIG. 4: Linear growth rate $\sigma$ of bed instability in a laminar river, versus the corresponding nondimensional wave number $k$. The fixed parameters values are $\beta=3.75, \gamma=1, S=0.0875$. The Froude number and aspect ratio are varied according to a straigh river widening (see section IV B and figure 5). Solid curves : $R=20.3$ and $F=3.94$. Short dashes : $R=35.0$ and $F=3.21$. Long dashes : $R=55.0$ and $F=2.71$. For each set $(R, F)$, the thick curve corresponds to the mode $n=1$, whereas the thin one correspond to the mode $n=2$. This behaviour provides an interpretation for the transition in patterns observed experimentally by [12].

high $n$ modes. Without it, the higher $n$, the higher $\sigma_{m}$. As in [10], we take $\gamma=1$ in the following. If $\beta=1$, that is if the sediment flux is proportional to the shear stress, then no instability ever appears (again $\sigma$ is always negative in that case). Instability may occur only if $\beta>1$. $\beta=3.75$ is choosen hereinafter as an illustrative case (see section II B).

Figure 4 illustrates the transition to bed instability as the aspect ratio is increased, for constant tilt and Froude number. A deep and narrow channel is stable, as for no values of $n$ and $k$ can $\sigma$ be negative. A shift to a larger aspect ratio value allows for the $n=1$ mode to be unstable. For a still wider channel, both $n=1$ and $n=2$ modes are unstable, but the latter grows faster. These transitions can be summarized in a three-dimensional phase diagram, with coordinates $R, F$ and $S$. A constant $S$ slice of this diagram is presented in figure 5. Even for null Froude number (and thus for null Reynolds number), may bars be unstable. This is a rather surprising feature, since this case allows to neglect the inertial terms in Saint-Venant equation. In other words, bars may develop in a purely viscous flow, which is impossible for dunes and ripples. Since a purely viscous flow can present no 
transverse recirculation, the above statement proves that neither turbulence nor recirculation are inherently linked to bars formation.

The diagram of figure 4 provides a crude interpretation for the ageing of laminar laboratory rivers. Let us consider for example the case of section III, for which the mean water level is fixed, while its bed and banks are freely eroded. If we assume a quasi-static evolution of the bed width so that the stability analysis for fixed wall can be roughly used, we can draw a schematic scenario for the river deformation. Thus, the tilt $S$ remains constant troughout the experiment whereas, in accordance with (20), the Froude number and aspect ratio evolve as follows:

$$
R \propto t^{2 /(\beta+2)}, \operatorname{Fr} \propto t^{3 /(2(\beta+2))} .
$$

This parameterized curve correspond to $F=F_{0}\left(R / R_{0}\right)^{3 / 4}$ in the stability diagram (the subscript 0 denotes initial conditions). In most cases this curve comes successively through the three stability domains of figure 5, allowing for the successive developpement of different bars modes. If the water output is conserved instead of the water level (this conditon is more common in experiments), the straight channel evolution is characterized by

$$
F=F_{0}\left(R / R_{0}\right)^{-3 / 8}
$$

Again, for realistic initial conditions $\left(R_{0}=20.3, F_{0}=3.21\right.$ in the experiment of [12]), the river undergoes different instability regimes as it ages. The three crosses drawn on figure 5 would then represent three different states of the same experiment, extrapolated from the initial condition using (36). The corresponding growth rate are plotted in Figure 4. When the highest growth rate of the first mode crosses zero, alternate bars appear, eventualy replaced by higher order modes.

If a threshold is introduced in the erosion law, the river eventually reaches an equilibrium state. The position of this equilibrium in the stability diagram is an indication about the instability patterns the river will preferentially develop. For instance, we may expect that a river will develop meanders if its equilibrium state lies in the domain where the $n=1$ mode is the most unstable one. 


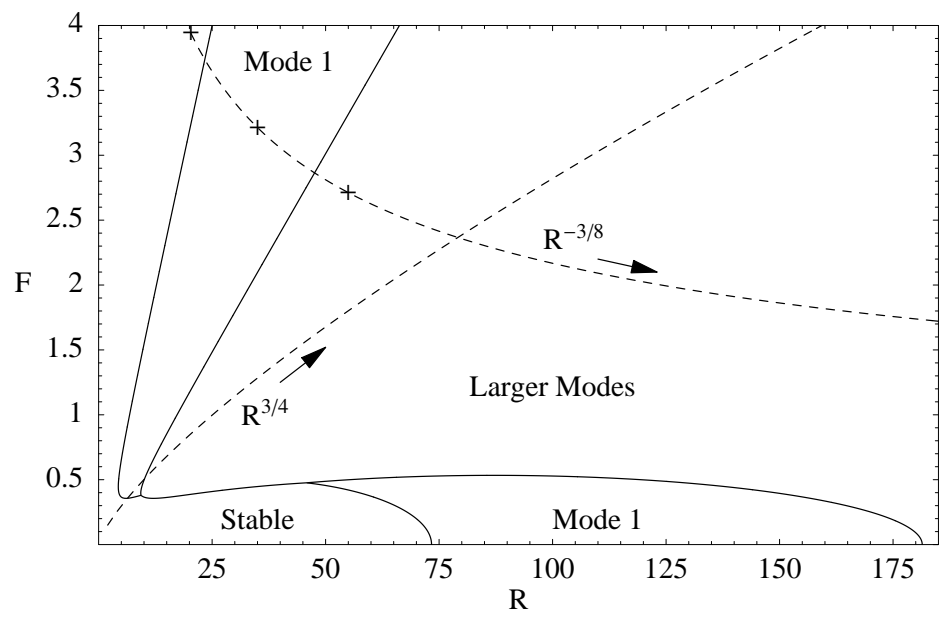

FIG. 5: Stability diagram for a laminar channel. The domains (separated by solid lines) are named after the most instable mode between $n=1$ and $n=2$. The parameters values are $\beta=3.75, \gamma=1$ and $S=0.0875$. The dashed lines represent the evolution of a straight river when the water level is imposed $\left(F=F_{0}\left(R / R_{0}\right)^{3 / 4}\right)$ or when the outflow is imposed $\left(F=F_{0}\left(R / R_{0}\right)^{-3 / 8}\right)$. The three cross correspond to the three cases presented in figure 4 .

\section{CONCLUSION}

The present paper demonstrates that the equations governing the evolution of laminar micro-rivers are very similar to their counterpart in the turbulent case. Experimental evidence of this similarity are collected in [11]. This results suggests that micro-rivers could facilitate the examination of some remaining difficulties of river morphodynamics, such as non-linearities or bank evolution. In a first attempt to develop viscous channel widening and stability theory, we presented a two dimensionnal shallow-water model. A very simplified analytical approach based on this model was sufficient to describe qualitatively the ageing process observed in some experiments. A diagram presenting the dominant unstable modes with respect to the channel tilt, Froude number and aspect ratio was obtained (figure 5), which shows a large domain of existence for the meandering mode $(n=1)$ at small (or even null) Froude number. This illustrate the sound difference beteween bars and dunes or ripples, which need inertia to grow.

The use of a fluid more viscous than water in experiments would allow to reach very low Froude numbers, while reducing the perturbating effect of capillarity. The consecutive re- 
duction of the Reynolds number would prevent recirculation, thus allowing the experimental separation between the effects of recirculation and bars instability.

The relaxation of the rigid banks hypothesis requires the development of bank erosion models, able to take avalanches into account. Such an improvement, associated with numerical simulation, would allow to test the laminar Saint-Venant theory aginst experiments in conditions closer to natural rivers ones.

It is our pleasure to thank B. Andreotti, P. Claudin, A. Fourrière, E. Lajeunesse, D. Lhuillier, L. Malverti, F. Métivier and G. Parker for inspiring and stimulating discussions.

[1] G. de Marsily, F. Delay, G. J., P. Renard, V. Teles, and S. Violette, Hydrogeol. J. 13, 161 (2005).

[2] A. Reynolds, J. Fluid Mech. 22, 113 (1965).

[3] E. Hansen, Basic Research Report 13, Copenhagen Technical University of Danemark (1967).

[4] R. A. Callander, J. Fluid Mech. 36, 465 (1969).

[5] G. Parker, J. Fluid Mech. 76, 457 (1976).

[6] F. Engelund and O. Skovgaard, J. Fluid Mech. 57, 289 (1973).

[7] S. Ikeda, G. Parker, and K. Saway, J. Fluid Mech. 112, 363 (1981).

[8] P. Blondeaux and G. Seminara, J. Fluid Mech. 157, 449 (1985).

[9] M. Colombini, G. Seminara, and M. Tubino, J. Fluid Mech. 181, 213 (1987).

[10] R. Schielen, A. Doelman, and H. de Swart, J. Fluid Mech. 252, 325 (1993).

[11] L. Malverti, G. Parker, L. Armstrong, P. Lancien, E. Lajeunesse, F. Métivier, S. Coleman, C. E. Smith, T. Davies, and A. Cantelli, Sedimentology (2006), (to appear).

[12] F. Metivier and P. Meunier, Journal of Hydrology 271, 22 (2003).

[13] B. Federici and C. Paola, Water Resour. Res. 39, 1162 (2003).

[14] F. Charru, H. Mouilleron, and O. Eiff, J. Fluid Mech. 519, 55 (2004).

[15] C. Ruyer-Quil and P. Manneville, Eur. Phys. J. pp. 357-369 (2000).

[16] F. Bouchut, A. Mangeney-Castelnau, B. Perthame, and J.-P. Vilotte, C. R. Acad. Sci. Paris pp. $531-536$ (2003).

[17] K. Blankaert and H. de Vriend, J. Fluid Mech. 498, 353 (2004).

[18] S. Ikeda and G. Parker, eds., River meandering (AGU Water Ressources Monograph, Wash- 
ington DC, 1989), vol. 12, chap. Observation on several recent theories of resonance and overdeepening in meandering channels, pp. 379-415.

[19] N. J. Balmforth and A. Provenzale, eds., Geomorphological Fluid Mechanics (Springer, 2001), chap. 16, pp. 394-402.

[20] L. v. Rijn, ed., Handbook of sediment transport by current and waves (Delft hydraulics, Delft, The Netherlands, 1989), vol. 12, pp. 379-415.

[21] P. Hersen, K. Andersen, H. Elbelrhiti, B. Andreotti, P. Claudin, and S. Douady, Physical Review 69 (2004).

[22] K. K. J. Kouakou and P.-Y. Lagrée, European journal of Mechanics B-Fluids 25, 348 (2006).

[23] A. Kovacs and G. Parker, J. Fluid Mech. 267, 153 (1994).

[24] G. Parker, J. Fluid Mech. 89, 109 (1978).

[25] G. Parker, J. Fluid Mech. 89, 127 (1978).

[26] A. Paquier and S. Khodashenas, Journal of Hydraulic Research 40 (2002).

[27] C. Stark, Geophysical Research Letters 33 (2006).

[28] L. Armstrong, Ph.D. thesis, Université Paris 7 Denis Diderot (2003).

[29] S. Ikeda, G. Parker, and Y. Kimura, Water Ressources Research 24, 713 (1988).

[30] T. Nakagawa, Sedimentology 30, 117 (1983).

[31] R. Giménez, Ph.D. thesis, Katholieke Universiteit Leuven (2003).

[32] Personal communication from E. Lajeunesse and F. Métivier

[33] This was observed during preliminary experiments carried out with L. Malverti and E. Lajeunesse.

[34] The idea of using highly viscous fluid in micro-rivers was first suggested by B. Andreotti. 